Original Article

\title{
Trajectories of sleep quality during the first three years after breast cancer diagnosis
}

\author{
Filipa Fontes a , Milton Severo ${ }^{\text {a, b }}$, Marta Gonçalves ${ }^{\text {a, c }}$, Susana Pereira ${ }^{\text {a, d }}$, Nuno Lunet ${ }^{\text {a, b, * }}$ \\ a ISPUP - EPIUnit, Universidade do Porto, Rua das Taipas, 4050-600 Porto, Portugal \\ ${ }^{\mathrm{b}}$ Departamento de Ciências da Saúde Pública e Forenses e Educação Médica, Faculdade de Medicina, Universidade do Porto, Al. Prof Hernâni Monteiro, \\ 4200-319 Porto, Portugal \\ ${ }^{\mathrm{c}}$ Centro de Medicina do Sono - Hospital CUF Porto, Estrada da Circunvalação, 4100-180 Porto, Portugal \\ d Instituto Português de Oncologia do Porto, Rua Dr. António Bernardino de Almeida, 4200-075 Porto, Portugal
}

\section{A R T I C L E I N F O}

\section{Article history:}

Received 18 December 2016

Received in revised form

9 February 2017

Accepted 2 March 2017

Available online 4 April 2017

\section{Keywords:}

Anxiety

Breast Neoplasm

Cluster analysis

Depression

Neuropathic pain

Sleep

\begin{abstract}
A B S T R A C T
Objective: To identify trajectories of sleep quality up to three years after breast cancer diagnosis and to assess differences in characteristics of patients across distinct trajectories.

Methods: A total of 458 breast cancer patients underwent a neurological evaluation before treatment and at one and three years after diagnosis. Clinical data were obtained throughout the follow-up. Anxiety and depression were evaluated at baseline, using the Hospital Anxiety and Depression Scale. In all sessions of follow-up, sleep quality was assessed using the Pittsburgh Sleep Quality Index. Model-based clustering was used to identify groups of patients with homogeneous variation in sleep quality.

Results: We identified three trajectories of variation in sleep quality, named "low" (LSQ), "medium" (MSQ), and "high sleep quality" (HSQ). Women in the HSQ trajectory presented good sleep quality during the three years. LSQ and MSQ trajectories were characterized by poor sleep quality during the whole period, although during the first year the latter depicted a significant deterioration of sleep quality and the former a significant improvement. Patients included in the LSQ trajectory were more likely to have clinically significant anxiety and depression at baseline. The two trajectories with worse sleep quality were associated with neuropathic pain three years after cancer diagnosis.

Conclusions: This study provides a model for describing the variation in sleep quality during the first three years after breast cancer diagnosis, based on three main trajectories. Further studies are needed understanding the heterogeneity of the individual trajectories within each of these major patterns of variation.
\end{abstract}

(c) 2017 Elsevier B.V. All rights reserved.

\section{Introduction}

Sleep disturbances are common among women with breast cancer, being estimated to affect up to $65 \%$ of patients [1-4], which is a greater proportion than observed in the general population $[5,6]$. In addition to factors that increase the vulnerability to develop sleep problems (eg, being female, older age, personal, and familial history of insomnia, co-occurrence of another psychiatric disorder) and to those that contribute to their maintenance over time (eg, maladaptive sleep behaviours, faulty beliefs about insomnia causes), stressful life events such as the diagnosis of an

\footnotetext{
* Corresponding author. Departamento de Ciências da Saúde Pública e Forenses e Educação Médica, Faculdade de Medicina, Universidade do Porto, Al. Prof Hernâni Monteiro, 4200-319 Porto, Portugal.

E-mail address: nlunet@med.up.pt (N. Lunet).
}

oncological disease or cancer treatments and their side-effects have been reported to act as precipitating factors, which may trigger development of sleep disturbance among cancer patients [7].

A systematic review assessing the impact of breast cancer treatments on sleep disturbances, concluded that women submitted to chemotherapy or radiotherapy reported higher levels of sleep disturbances than those not undergoing these treatments; however, evidence from prospective studies evaluating patients before and after treatment was scarce [8]. In fact, it has been noted that a high proportion of women already presented higher levels of sleep disturbance prior to treatments $[9,10]$ whereas some other patients with insomnia reported that it began months after diagnosis [11], highlighting the need for a comprehensive evaluation of different longitudinal patterns of sleep quality among women with breast cancer. 
Neurological side effects of breast cancer treatments are frequent during the first year after cancer diagnosis [12], and affect more than half of the women treated for breast cancer after three years [13]. Although neuropathic pain (NP), chemotherapy-induced peripheral neuropathy (CIPN) and cognitive impairment are among the most frequently described side effects, there is scarce information about their contribution to different trajectories of sleep quality.

Therefore, the purpose of this study was to identify trajectories of sleep quality during the first three years after breast cancer treatment and to assess differences in sociodemographic and clinical characteristics of the patients across distinct trajectories of sleep quality.

\section{Material and methods}

This study is part of a larger prospective cohort study assessing neurological complications of breast cancer and its treatments among women with newly diagnosed breast cancer, followed for three years. The study protocol has been described in detail elsewhere [14].

\subsection{Participants and setting}

Participants were consecutively recruited in 2012, among those proposed for surgery, aged 18 years or older, admitted to the Breast Clinic of the Portuguese Institute of Oncology of Porto (IPO-Porto), Portugal. Briefly, we excluded those treated with chemotherapy and/or radiotherapy in the chest or axillary areas for other primary cancer, those that had received any treatment for breast cancer before, and those with a high probability of cognitive impairment [score lower than 17, or lower than 16 for women over 65 years, in the Montreal Cognitive Assessment (MoCA) [15]]. A total of 506 participants were enrolled in the cohort and underwent a baseline evaluation before treatment.

\subsection{Procedures and measures}

Participants were evaluated, including a neurological assessment, at baseline (before any treatment) and at one and three years after enrolment. The median time [percentile 25-percentile 75 (P25-P75)] between diagnosis (date of first histological confirmation) and the baseline evaluation was 24 days (18-32).

At baseline, sociodemographic data were collected using a structured questionnaire, and clinical records were reviewed for cancer stage and menopausal status. Cancer stage was classified according to the American Joint Committee on Cancer Staging Manual [16]. When menopausal status was not specified, all women older than 60 years, women who underwent a bilateral ovariectomy and those with an intact uterus and being amenorrheic for at least one year prior to the diagnosis of breast cancer were classified as postmenopausal, or otherwise as premenopausal [17].

At one and three years after enrolment, clinical records were examined for breast cancer treatments and the occurrence of cancer relapse. In addition, the presence of neurological complications affecting the patients at one and/or three years after cancer diagnosis, namely NP and CIPN was recorded. NP was considered probable, according to the classification system adopted by the International Association for the Study of Pain, when pain distribution was neuroanatomically plausible and history was suggestive of relevant lesions or diseases affecting the somatosensory system, plus negative or positive sensory signs in neurological examination, confined to the innervation territory of the injury nervous structure [18]. We considered NP as prevalent in each of the evaluations if it was present in the last $24 \mathrm{~h}$, in the breast, chest wall, axilla, or medial upper arm on the affected side, donor region of breast reconstruction, or in hands/feet (secondary to CIPN). CIPN was defined as peripheral neuropathy beginning after chemotherapy. Among patients with preexisting peripheral neuropathy at baseline, CIPN was considered to have occurred only if there was a worsening of neuropathy after the beginning of chemotherapy.

Each patient was evaluated with MoCA (range: 0-30) at baseline, and at one and three years. In each evaluation, cognitive impairment was considered present, when the corresponding MoCA score was at least 2.0 standard deviations below age- and education-adjusted cut-offs for possible cognitive impairment [15].

The Hospital Anxiety and Depression Scale (HADS) [19] was used to evaluate anxiety and depression at baseline. The HADS consists of 14 items, with two seven-item subscales assessing anxiety and depression in the past week (range: 0-21 for each subscale); a score greater than or equal to 11 for each of them was considered to be indicative of clinically significant anxiety and/or depression, as applicable [19].

The Pittsburgh Sleep Quality Index (PSQI) was used to evaluate sleep quality at baseline, and at one and three years [20]. The PSQI includes 19 items measuring seven components, including subjective sleep quality, sleep latency, sleep duration, habitual sleep efficiency, sleep disturbances, use of sleep medication and daytime dysfunction. Components (range: $0-3$ ) are summed to provide a global score designed to measured sleep quality over the past month (range: 0-21); a score greater than five indicates poor sleep quality [20].

\subsection{Statistical analysis}

From the 506 participants who underwent a baseline evaluation, 31 were lost to follow-up until the third year (11 patients died, 10 abandoned the study, six could not be contacted, two were transferred to another hospital and two were considered unable to cooperate by the neurologist). For the purposes of this study, we excluded those with cancer stage IV at baseline $(\mathrm{N}=3)$, patients with cancer relapse until the third year of follow-up $(\mathrm{N}=12)$ and those with missing information on sleep quality $(\mathrm{N}=2)$. Therefore, a total of 458 participants were included in the present analysis. The patients not included $(\mathrm{N}=48)$ were not significantly different (participants included vs. participants not included) regarding age ( $<55$ years: $52.8 \%$ vs. $52.1 \%$; $P=0.921$ ), education ( $\leq 4$ years: $42.6 \%$ vs. $43.5 \% ; \mathrm{P}=0.968$ ) and presence of anxiety (39.0\% vs. $29.2 \%$; $\mathrm{P}=0.184$ ), depression (7.9\% vs. $10.4 \%$; $\mathrm{P}=0.537$ ) and poor sleep quality (60.0\% vs. $61.7 \% ; \mathrm{P}=0.825$ ) at baseline. However, they had more frequently more advanced cancer stages at baseline (cancer stage III: $14.2 \%$ vs. $29.2 \%$; $\mathrm{P}=0.017$ ).

Model-based clustering [21] was used to identify groups of patients that share similar time trends in the PSQI global score, while distinguishing them from other groups of patients homogeneous regarding the variation in sleep quality. According to this method, the data are assumed to have a multivariate normal distribution, parameterised by their mean and covariance, generated by clusters. The geometric features (orientation, volume, and shape) of the distribution are estimated from the data, and can be allowed to vary between clusters, or constrained to be the same for all clusters [22]. The most appropriate model was considered allowing for the most homogenous grouping of patients regarding their patterns of sleep variation, as assessed by visual inspection, would be selected among those with the lowest value of Bayesian Information Criterion.

Sample characteristics are presented as counts and proportions for categorical variables and median and P25-P75 for quantitative variables with markedly asymmetrical distribution. The mean PSQI score between the baseline and the one year follow-up evaluations 
and between the one and the three year follow-up evaluations were compared using the Student's' $t$ test for paired samples, for each of the trajectories identified.

Odds ratio (OR) and the corresponding 95\% confidence intervals $(95 \% \mathrm{CI})$ for the association between different characteristics of the patients, including cancer treatments and neurological complications, and trajectories of sleep quality up to three years after breast cancer diagnosis, were computed using multinomial logistic regression. Variables included in each model are those known to be associated with the exposure and outcome of interest, without being an intermediate steep in this relation, as supported by scientific literature, and are described as footnotes of Tables 1 and 2.

Statistical analysis were conducted using R, version 3.0.1 (R Core Team, Vienna, Austria) and STATA ${ }^{\circledR}$, version 11.2 (StataCorp, College Station, TX, USA).

\subsection{Ethics}

The study was approved by the Ethics Committee of the IPOPorto (CES 406/011, CES 99/014, and CES 290/014) and by the Portuguese Data Protection Authority (Ref. 9469/2012 and Ref 8601/ 2014). All participants provided written informed consent.

\section{Results}

At baseline, more than half of the women were less than 55 years of age (52.8\%) and more than two thirds was less than 10 years of education (71.2\%). Most of them were diagnosed with cancer stage 0 or I (55.0\%). A total of $84.3 \%, 73.1 \%$, and $59.4 \%$ received endocrine therapy, radiotherapy, and chemotherapy, in addition to surgical treatment.

Table 1

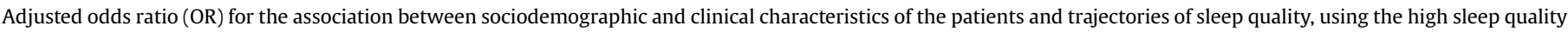
trajectory as reference.

\begin{tabular}{|c|c|c|c|c|c|}
\hline & \multirow{2}{*}{$\frac{\text { High sleep quality (HSQ) }}{\mathrm{N}(\%)}$} & \multicolumn{2}{|c|}{ Medium sleep quality (MSQ) } & \multicolumn{2}{|c|}{ Low sleep quality (LSQ) } \\
\hline & & $\mathrm{N}(\%)$ & OR $(95 \% \mathrm{CI})$ & $\mathrm{N}(\%)$ & OR $(95 \% \mathrm{CI})$ \\
\hline \multicolumn{6}{|l|}{ Age (years) } \\
\hline$<55$ & $44(18.2)$ & $123(50.8)$ & 1 (ref.) & $75(31.0)$ & 1 (ref.) \\
\hline$\geq 55$ & $29(13.4)$ & $121(56.0)$ & $1.49(0.88-2.54)$ & $66(30.6)$ & $1.34(0.75-2.67)$ \\
\hline \multicolumn{6}{|l|}{ Education (years) } \\
\hline$\leq 4$ & $25(12.8)$ & $103(52.8)$ & 1 (ref.) & $67(34.4)$ & 1 (ref.) \\
\hline$\overline{5}-9$ & $23(17.6)$ & $68(51.9)$ & $0.82(0.41-1.63)^{f}$ & $40(30.5)$ & $0.67(0.32-1.40)^{\mathrm{f}}$ \\
\hline$\geq 10$ & $25(18.9)$ & $73(55.3)$ & $0.81(0.41-1.60)^{\mathrm{f}}$ & $34(27.8)$ & $0.52(0.25-1.10)^{\mathrm{f}}$ \\
\hline \multicolumn{6}{|c|}{ Anxiety at baseline ${ }^{\mathrm{b}}\left[\mathrm{N}=457^{\mathrm{a}}\right]$} \\
\hline No & $55(19.7)$ & $162(58.1)$ & 1 (ref.) & $62(22.2)$ & 1 (ref.) \\
\hline Yes & $18(10.1)$ & $81(45.5)$ & $1.56(0.86-2.83)^{g}$ & $79(44.4)$ & $3.93(2.09-7.39)^{\mathrm{g}}$ \\
\hline \multicolumn{6}{|c|}{ Depression at baseline ${ }^{c}$} \\
\hline No & $72(17.1)$ & $232(55.0)$ & 1 (ref.) & $118(28.0)$ & 1 (ref.) \\
\hline Yes & $1(2.8)$ & $12(33.3)$ & $3.69(0.47-29.0)^{g}$ & $23(63.9)$ & $14.6(1.9-110.9)^{g}$ \\
\hline \multicolumn{6}{|c|}{ Cognitive impairment at baseline } \\
\hline No & $65(15.5)$ & $224(53.3)$ & 1 (ref.) & $131(31.2)$ & 1 (ref.) \\
\hline Yes & $8(21.0)$ & $20(52.6)$ & $0.82(0.33-2.02)^{\mathrm{h}}$ & $10(26.3)$ & $0.72(0.25-2.08)^{\mathrm{h}}$ \\
\hline \multicolumn{6}{|c|}{ Menopausal status at baseline $\left[\mathrm{N}=441^{\mathrm{a}}\right]$} \\
\hline Premenopausal & $40(21.0)$ & $92(48.4)$ & 1 (ref.) & $58(30.5)$ & 1 (ref.) \\
\hline Postmenopausal & $31(12.4)$ & $140(55.8)$ & $2.53(0.88-7.27)^{\mathrm{f}}$ & $80(31.9)$ & $2.64(0.87-7.98)^{f}$ \\
\hline \multicolumn{6}{|l|}{ Cancer stage } \\
\hline 0 (DCIS)/I & $39(15.5)$ & $133(52.8)$ & 1 (ref.) & $80(31.8)$ & 1 (ref.) \\
\hline II & $25(17.7)$ & $76(53.9)$ & $0.67(0.32-1.42)^{\mathrm{i}}$ & $40(28.4)$ & $0.83(0.43-1.58)^{\mathrm{i}}$ \\
\hline III & $9(13.8)$ & $35(52.8)$ & $0.53(0.25-1.11)^{\mathrm{i}}$ & $21(32.3)$ & $1.20(0.50-2.91)^{\mathrm{i}}$ \\
\hline \multicolumn{6}{|l|}{ Breast surgery $^{\mathrm{d}}$} \\
\hline Breast-conserving & $40(17.2)$ & $118(50.6)$ & 1 (ref.) & $75(32.2)$ & 1 (ref.) \\
\hline Mastectomy & $33(14.7)$ & $126(56.0)$ & $1.33(0.76-2.33)^{g}$ & $66(29.3)$ & $1.11(0.60-2.02)^{g}$ \\
\hline \multicolumn{6}{|l|}{ Axillary surgery } \\
\hline None/SLNB & $51(16.6)$ & $162(52.6)$ & 1 (ref.) & $95(30.8)$ & 1 (ref.) \\
\hline ALND & $22(14.7)$ & $82(54.7)$ & $1.35(0.60-3.02)^{g}$ & $46(30.7)$ & $1.33(0.55-3.20)^{g}$ \\
\hline \multicolumn{6}{|l|}{ Chemotherapy } \\
\hline No & $26(14.0)$ & $92(49.5)$ & 1 (ref.) & $68(36.6)$ & 1 (ref.) \\
\hline Yes & $47(17.3)$ & $152(55.9)$ & $0.98(0.51-1.90)^{g}$ & $73(26.8)$ & $0.53(0.26-1.10)^{g}$ \\
\hline \multicolumn{6}{|c|}{ Radiotherapy (internal and/or external) } \\
\hline No & $14(14.2)$ & $66(55.0)$ & 1 (ref.) & $37(30.8)$ & 1 (ref.) \\
\hline Yes & $56(16.6)$ & $178(52.7)$ & $1.00(0.39-2.57)^{\mathrm{j}}$ & $104(30.8)$ & $0.81(0.29-2.26)^{j}$ \\
\hline \multicolumn{6}{|l|}{ Endocrine therapy } \\
\hline No & $10(13.9)$ & $42(58.3)$ & 1 (ref.) & $20(27.8)$ & 1 (ref.) \\
\hline Yes & $63(16.3)$ & $202(52.3)$ & $0.97(0.43-2.16)^{\mathrm{k}}$ & $121(31.4)$ & $1.05(0.44-2.52)^{\mathrm{k}}$ \\
\hline \multicolumn{6}{|l|}{ Immunotherapy } \\
\hline No & $61(15.3)$ & $211(52.9)$ & 1 (ref.) & $127(31.8)$ & 1 (ref.) \\
\hline Yes & $12(30.3)$ & $33(55.9)$ & $0.82(0.39-1.69)^{g}$ & $14(23.7)$ & $0.59(0.25-1.36)^{g}$ \\
\hline
\end{tabular}

ALND, Axillary lymph node dissection; DCIS, Ductal carcinoma in situ; OR, Odds radio; SLNB, Sentinel lymph node biopsy; 95\%CI, 95\% confidence interval.

a $\mathrm{N}<458$ due to missing data.

b Defined as a score greater than or equal to 11 in the anxiety subscale of the Hospital Anxiety and Depression Scale.

c Defined as a score greater than or equal to 11 in the depression subscale of the Hospital Anxiety and Depression Scale.

d Patients who had both mastectomy and breast-conserving surgery are reported as mastectomy.

e Patients who had both ALND and SLNB are reported as ALND.

f Adjusted for age.

g Adjusted for age, education and cancer stage.

h Adjusted for age, education, cancer stage, anxiety at baseline, depression at baseline, chemotherapy and endocrine therapy.

i Adjusted for age and education.

j Adjusted for age, education, cancer stage, and breast and axillary surgery.

$\mathrm{k}$ Adjusted for age, education, cancer stage and menopausal status. 
Table 2

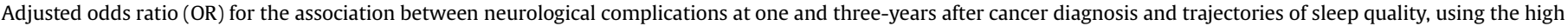
sleep quality trajectory as reference.

\begin{tabular}{|c|c|c|c|c|c|}
\hline & \multirow{2}{*}{$\frac{\text { High sleep quality (HSQ) }}{\mathrm{N}(\%)}$} & \multicolumn{2}{|c|}{ Medium sleep quality (MSQ) } & \multicolumn{2}{|c|}{ Low sleep quality (LSQ) } \\
\hline & & $\mathrm{N}(\%)$ & OR $(95 \% \mathrm{CI})$ & $\mathrm{N}(\%)$ & OR $(95 \% \mathrm{CI})$ \\
\hline \multicolumn{6}{|c|}{ Neuropathic pain at one-year } \\
\hline No & $64(17.6)$ & $188(51.8)$ & 1 (ref.) & $111(30.6)$ & 1 (ref.) \\
\hline Yes & $9(9.5)$ & $56(59.0)$ & $2.01(0.89-4.51)^{\mathrm{a}}$ & $30(31.6)$ & $1.23(0.51-2.99)^{\mathrm{a}}$ \\
\hline \multicolumn{6}{|c|}{ Neuropathic pain at three-years } \\
\hline No & $68(19.4)$ & $187(53.4)$ & 1 (ref.) & $95(27.1)$ & 1 (ref.) \\
\hline Yes & $5(4.6)$ & $57(52.8)$ & $4.12(1.55-10.94)^{\mathrm{a}}$ & $46(42.6)$ & $5.49(1.99-15.12)^{\mathrm{a}}$ \\
\hline \multicolumn{6}{|c|}{ CIPN at one-year } \\
\hline No & $66(16.7)$ & 209 (52.9) & 1 (ref.) & $120(30.4)$ & 1 (ref.) \\
\hline Yes & $7(11.1)$ & $35(55.6)$ & $1.68(0.67-4.17)^{\mathrm{b}}$ & $21(33.3)$ & $2.13(0.79-5.79)^{\mathrm{b}}$ \\
\hline \multicolumn{6}{|c|}{ CIPN at three-years } \\
\hline No & $67(14.5)$ & $217(53.3)$ & 1 (ref.) & $123(30.2)$ & 1 (ref.) \\
\hline Yes & $6(11.8)$ & $27(52.9)$ & $1.40(0.53-3.71)^{\mathrm{b}}$ & $18(35.3)$ & $2.05(0.71-5.91)^{\mathrm{b}}$ \\
\hline \multicolumn{6}{|c|}{ Cognitive impairment at one-year } \\
\hline No & $68(16.0)$ & $227(53.4)$ & 1 (ref.) & $130(30.6)$ & 1 (ref.) \\
\hline Yes & $5(15.2)$ & $17(51.5)$ & $1.11(0.38-3.20)^{\mathrm{c}}$ & $11(33.3)$ & $1.21(0.37-3.89)^{\mathrm{c}}$ \\
\hline \multicolumn{6}{|c|}{ Cognitive impairment at three-years } \\
\hline No & $68(16.2)$ & $223(53.0)$ & 1 (ref.) & $130(30.9)$ & 1 (ref.) \\
\hline Yes & $5(13.5)$ & $21(56.8)$ & $1.22(0.44-3.41)^{c}$ & $11(29.7)$ & $0.87(0.28-2.76)^{c}$ \\
\hline
\end{tabular}

CIPN, Chemotherapy-induced peripheral neuropathy.

a Adjusted for age, education, cancer stage, anxiety at baseline, depression at baseline, chemotherapy, breast and axillary surgery and radiotherapy.

b Adjusted for age, education, cancer stage, anxiety at baseline, depression at baseline and chemotherapy.

c Adjusted for age, education, cancer stage, anxiety at baseline, depression at baseline, chemotherapy and endocrine therapy.

Three distinct clusters of patients with similar variation in sleep quality over a period of three years after breast cancer diagnosis were identified (Fig. 1). Trajectory 1 (141 patients) and trajectory 2 (244 patients), labelled as "low" (LSQ) and "medium sleep quality" (MSQ), respectively, though presenting significant differences between them, both are characterized by mean values of poor sleep quality (PSQI global score $>5$ ) during the whole period. However, while in the former there was an improvement in sleep quality $(P<0.001)$, the latter is characterized by a deterioration in sleep quality $(\mathrm{P}<0.001)$ during the first year. After the one-year followup evaluation, there was a levelling-off in sleep quality in both trajectories. Trajectory 3 (73 patients), labelled as "high sleep quality" (HSQ) presents mean values of good sleep quality (PSQI global score $\leq 5$ ) and a stable trend in PSQI global score during the whole follow-up.

The PSQI components scores in the three moments of evaluation, according to the different trajectories of sleep quality, are depicted in Fig. 2. The LSQ trajectory is characterized by higher mean values of each PSQI components than those found in the

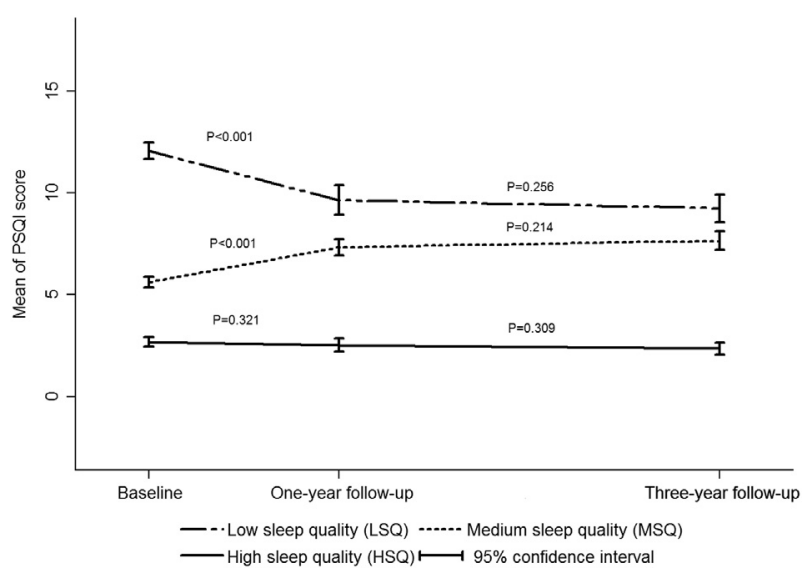

Fig. 1. Means and the corresponding 95\% confidence intervals of the Pittsburgh Sleep Quality Index (PSQI) score for each trajectory identified. PSQI, Pittsburgh Sleep Quality Index. Higher PSQI scores correspond to a worse sleep quality. remaining trajectories, in all the moments of follow-up, and by a decrease in sleep latency and daytime dysfunction and by an increase in sleep duration and habitual sleep efficiency, between baseline and the one-year follow-up evaluation. The HSQ trajectory presented the lowest mean scores in all PSQI components; sleep disturbances were the major contributor to overall PSQI score, with at least two-times higher values than other components of the same trajectory. In the MSQ trajectory there was a more similar distribution between PSQI components scores in the three evaluations, and a decrease in habitual sleep efficiency and an increase in the use of sleep medication between baseline and the one-year follow-up evaluation.

Table 1 presents results for the association between sociodemographic and clinical characteristics of patients and trajectories of sleep quality. Anxiety and depression prior to the beginning of treatments were significantly more frequent among patients in LSQ than in those in HSQ.

There were no statistically significant differences between treatments performed during the first year of follow-up across the different trajectories of sleep quality (Table 1). However, those in the LSQ and in the MSQ trajectories were more likely to present NP three years after cancer diagnosis (Table 2).

\section{Discussion}

Our study identified three main trajectories of variation in sleep quality during three years after breast cancer diagnosis. Despite the LSQ and the MSQ trajectories presented values of poor sleep quality during the whole follow-up, the latter is characterized by a deterioration in sleep quality and the former by an improvement in sleep quality during the first year. The HSQ trajectory is characterized by good sleep quality during the whole period. Patients included in the LSQ trajectory were more likely to present anxiety and depression prior to treatments. The two trajectories with poor sleep quality over time presented more frequently NP three years after cancer diagnosis.

To our knowledge, only one previous study identified subgroups of breast cancer patients based on changes in sleep quality over time, using a model-based approach [23]. Using growth mixture 


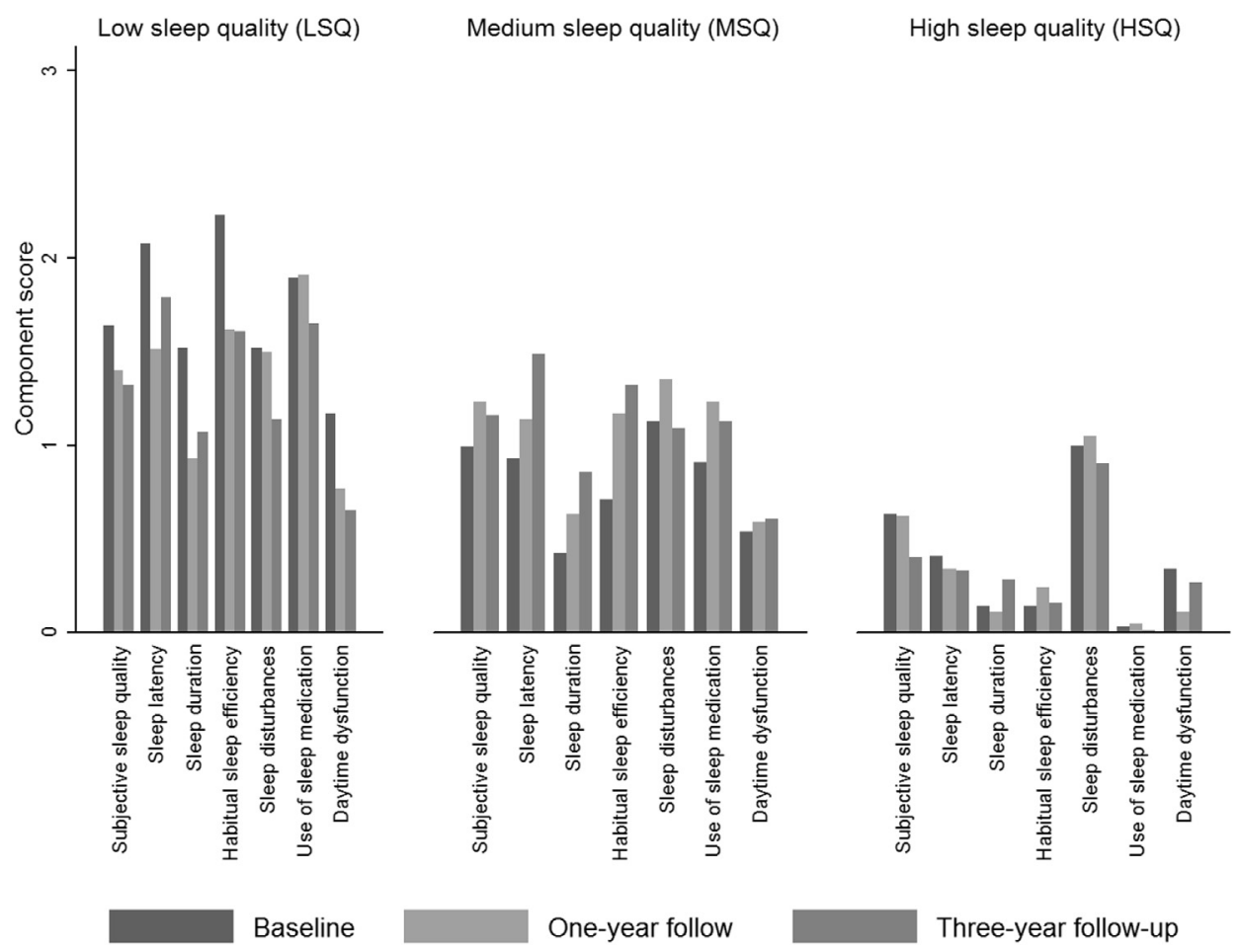

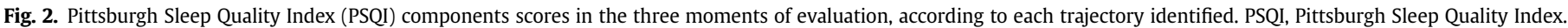

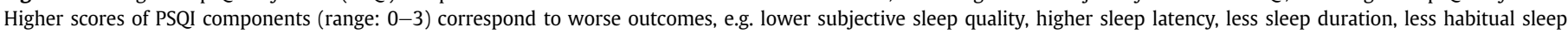
efficiency, higher sleep disturbances, higher use of sleep medication and higher daytime dysfunction.

modelling, Van Onselen et al. described three distinct sleep disturbance trajectories in a sample of approximately 400 women enrolled prior to surgery and followed during six months; their study yielded a "high sustained class" and a "low sustained class" that had high and low levels of sleep disturbance prior to surgery that persisted for six months, respectively, and a "decreasing class" that had high levels of sleep disturbance prior to surgery that decreased over time, although the latter was identified in only 21 patients [23]. Despite direct comparisons between the two studies are hampered by the use of different methods to evaluate sleep quality and to summarize the trends, and by the use of different follow-up periods and moments of sleep assessment, both studies identified one trajectory with levels of good sleep quality constant over time and another one with a trend toward better sleep quality during the first year. The longer follow-up of our analysis adds to previous research the identification of trajectories during a period of three years.

Other prospective studies have evaluated sleep quality in breast cancer patients in different periods after cancer diagnosis [24,25]. In a study assessing women scheduled to receive neoadjuvant or adjuvant chemotherapy for breast cancer, Ancoli-Israel et al. showed that compared to non-cancer controls breast cancer patients had significantly worse scores for sleep quality, fatigue, depressive symptoms and quality of life, at baseline, during chemotherapy and after one year; among breast cancer patients, sleep quality returned to baseline levels at one year [24]. Using data from the same cohort of patients, Liu et al. reported that breast cancer patients with a higher pre-treatment symptom cluster index (worse sleep, more fatigue and more depressive symptoms) were also more symptomatic during chemotherapy, when compared to those with fewer symptoms before treatment [25]. Despite methodological differences, our findings are in accordance with the previous results, since in our study the patients' sleep quality trajectory was largely influence by sleep quality at baseline and there was a statistically significant relation between sleep quality over time and the presence of anxiety and depression at baseline

Psychiatric disorders have been described as predisposing factors for insomnia in the general population [26] and in breast cancer patients $[2,27]$. On the other hand, insomnia symptoms are among the diagnostic criteria and risk factors for several psychiatric disorders [28]. Although it was not surprising to find a higher frequency of anxiety and depression in trajectories with worse sleep quality prior to treatments and over time, our strategy of data analysis precludes the assumption of a direction for the association between anxiety and depression, and sleep quality. The trend toward an improvement in sleep quality during the first year of follow-up, among those in the LSQ trajectory, may be explained by the treatment of some of the patients with greater scores of anxiety and depression prior to treatments, and its subsequent impact on sleep quality one year after enrolment. Unfortunately, the absence of data regarding pharmacological treatments for anxiety and depression, at baseline and during the follow-up, did not allow us to test this hypothesis.

Although not statistically significant, there was a higher proportion of postmenopausal women in trajectories with poor sleep quality at baseline. This is consistent with previous knowledge that menopausal vasomotor symptoms (including hot flashes) are associated with disrupted sleep [27,29]. However, a study that evaluated the effect of menopausal symptoms on sleep quality in breast cancer patients, using both objective and subjective measures, found that women who were premenopausal before and after chemotherapy had worse sleep than those who were either premenopausal prior to chemotherapy and became perimenopausal after chemotherapy or were postmenopausal [30]; the authors hypothesized that other physical or psychological variables not evaluated in their study, such as the prior exposure to menopausal symptoms, could have contributed to their results [30]. In our study, the absence of data regarding the potential modification 
of the menopausal status of some of the breast cancer patients during treatments trajectory, either naturally or as a consequence of cancer treatment, did not allow us to infer about the role of this variable on the change of sleep quality pattern, mainly during the first year of follow-up.

Previous studies have supported the strong relation between fatigue and sleep, reporting that the two symptoms often co-occur with depression as part of a symptom cluster in breast cancer patients [31,32]. Ho et al. investigated the relation between the three symptoms showing moderate to strong associations, prior to and following chemotherapy for breast cancer [31]. In another study, Berger et al. examined patterns of circadian rhythms and their relationship with fatigue, anxiety and depression, and concluded that disrupted patterns of circadian rhythms were associated with distressing fatigue and depressive symptoms during chemotherapy and at recovery [32]. Therefore, although fatigue was not evaluated in the present study, it may have also contributed to the sleep trajectories among these patients.

$\mathrm{NP}$ is a frequent complication of both surgical and adjuvant treatments for breast cancer $[33,34]$. In or study, both the LSQ and the MSQ trajectories, were more likely to include patients with NP at the three-year follow-up. It has been described that the context in which a painful stimulus occurs affects how patients perceive it and that psychological factors such as depression, somatization, poor coping skills, and social stressors can predict the development of chronic pain [35]. In addition, in a previous analysis of data from the same cohort of patients, we described that NP was less severe among women presenting this condition at the one-year follow-up evaluation than those with NP three years after cancer diagnosis [13], which could at least in part, explain the absence of statistically significant differences in the proportion of NP at one-year across different trajectories.

The major strengths of our study are the longitudinal analysis of sleep quality, with an evaluation prior to treatments and the much longer follow-up than the previous study analysing subgroups of breast cancer patients based on changes in sleep quality over time. However, some limitations need to be acknowledged. As we did not evaluate sleep quality before cancer diagnosis, the proportion of patients for which poor sleep quality is a consequence of the diagnosis of an oncological disease is unknown. Also, the assessment of a cohort with a large number of participants precludes the use of objective measures of sleep to corroborate the patients' self-reports. Nevertheless, PSQI is one of the measures recommended to assess sleep or insomnia symptoms in epidemiological studies and has demonstrated good psychometric properties [36]. Finally, NP and the remaining neurological complications had only clinical diagnosis, which may limit the accuracy of the information regarding these disorders. Nevertheless, our results reflect findings in usual clinical practice, since all patients were treated according to the usual practice of a major oncological hospital.

\section{Conclusions}

The present study provides a model for describing the variation in sleep quality during three years after cancer diagnosis, based in three main trajectories. Our results show that a high proportion of women already presented poor sleep quality prior to treatments, which persisted during three years. In addition, there was a relation between trajectories of worse sleep quality during this period and psychiatric disorders at baseline and NP at three years. These results emphasize the importance of monitoring sleep quality prior to the onset of cancer treatment, and reinforce the necessity of management of comorbidities for a comprehensive treatment of sleep disorders among breast cancer patients. Further studies are needed to understand the heterogeneity of the individual trajectories within each of these major patterns of variation, and their associated factors.

\section{Funding}

This study was funded by FEDER through the Operational Programme Competitiveness and Internationalization (POCI-01-0145FEDER-016867) and national funding from the Foundation for Science and Technology - FCT (Portuguese Ministry of Science, Technology and Higher Education) (PTDC/DTP-EPI/7283/2014) under the Unidade de Investigação em Epidemiologia - Instituto de Saúde Pública da Universidade do Porto (EPIUnit) (POCI-01-0145FEDER-006862; Ref.UID/DTP/04750/2013); the PhD Grant SFRH/ BD/92630/2013 (Filipa Fontes) co-funded by the FCT and the POPH/ FSE Program. Data management activities up to the first year of follow-up were supported by the Chair on Pain Medicine of the Faculty of Medicine, University of Porto and by the Grünenthal Foundation - Portugal.

\section{Conflict of interest}

The ICMJE Uniform Disclosure Form for Potential Conflicts of Interest associated with this article can be viewed by clicking on the following link: http://dx.doi.org/10.1016/j.sleep.2017.03.022.

\section{References}

[1] Otte JL, Carpenter JS, Russell KM, et al. Prevalence, severity, and correlates of sleep-wake disturbances in long-term breast cancer survivors. J Pain Symptom Manage 2010;39(3):535-47.

[2] Desai K, Mao JJ, Su I, et al. Prevalence and risk factors for insomnia among breast cancer patients on aromatase inhibitors. Support Care Cance 2013;21(1):43-51.

[3] Bardwell WA, Profant J, Casden DR, et al. The relative importance of specific risk factors for insomnia in women treated for early-stage breast cancer. Psychooncology 2008;17(1):9-18.

[4] Fortner BV, Stepanski EJ, Wang SC, et al. Sleep and quality of life in breast cancer patients. J Pain Symptom Manage 2002;24(5):471-80.

[5] Carlson LE, Campbell TS, Garland SN, et al. Associations among salivary cortisol, melatonin, catecholamines, sleep quality and stress in women with breast cancer and healthy controls. J Behav Med 2007;30(1):45-58.

[6] Carpenter JS, Elam JL, Ridner SH, et al. Sleep, fatigue, and depressive symptoms in breast cancer survivors and matched healthy women experiencing hot flashes. Oncol Nurs Forum 2004;31(3):591-5598.

[7] Savard J, Morin CM. Insomnia in the context of cancer: a review of a neglected problem. J Clin Oncol 2001;19(3):895-908.

[8] Costa AR, Fontes F, Pereira S, et al. Impact of breast cancer treatments on sleep disturbances - a systematic review. Breast 2014;23(6):697-709.

[9] Denieffe S, Cowman S, Gooney M. Symptoms, clusters and quality of life prior to surgery for breast cancer. J Clin Nurs 2014:23(17-18):2491-502.

[10] Savard J, Villa J, Ivers $H$, et al. Prevalence, natural course, and risk factors of insomnia comorbid with cancer over a 2-month period. J Clin Oncol 2009;27(31):5233-9.

[11] Savard J, Simard S, Blanchet J, et al. Prevalence, clinical characteristics, and risk factors for insomnia in the context of breast cancer. Sleep 2001;24(5):583-90.

[12] Pereira S, Fontes F, Sonin T, et al. Neurological complications of breast cancer: a prospective cohort study. Breast 2015;24(5):582-7.

[13] Fontes F, Pereira S, Castro-Lopes JM, et al. A prospective study on the neurological complications of breast cancer and its treatment: updated analysis three years after cancer diagnosis. Breast 2016;29:31-8.

[14] Pereira S, Fontes F, Sonin T, et al. Neurological complications of breast cancer: study protocol of a prospective cohort study. BMJ Open 2014;4(10):e006301.

[15] Freitas S, Simoes MR, Alves L, et al. Montreal cognitive assessment (MoCA): normative study for the Portuguese population. J Clin Exp Neuropsychol 2011;33(9):989-96.

[16] Edge S, Byrd D, Compton C, et al. AJCC cancer staging manual. 7th ed. New York: Springer; 2010.

[17] De Vos FY, van Laarhoven HW, Laven JS, et al. Menopausal status and adjuvant hormonal therapy for breast cancer patients: a practical guideline. Crit Rev Oncol Hematol 2012;84(2):252-60.

[18] Haanpaa M, Attal N, Backonja M, et al. NeuPSIG guidelines on neuropathic pain assessment. Pain 2011;152(1):14-27.

[19] Zigmond AS, Snaith RP. The hospital anxiety and depression scale. Acta Psychiatr Scand 1983;67(6):361-70. 
[20] Buysse DJ, Reynolds 3rd CF, Monk TH, et al. The Pittsburgh Sleep Quality Index: a new instrument for psychiatric practice and research. Psychiatry Res 1989;28(2):193-213.

[21] Fraley C, Raftery AE. Model-based clustering, discriminant analysis, and density estimation. J Am Stat Assoc 2002;97(458):611-31.

[22] Fraley C, Raftery AE, Murphy TB, et al. Mclust version 4 for R: normal mixture modeling for model-based clustering, classification, and density estimation. Technical Report No. 597. Department of Statistics, University of Washington; 2012.

[23] Van Onselen C, Cooper BA, Lee K, et al. Identification of distinct subgroups of breast cancer patients based on self-reported changes in sleep disturbance. Support Care Cancer 2012;20(10):2611-9.

[24] Ancoli-Israel S, Liu L, Rissling M, et al. Sleep, fatigue, depression, and circadian activity rhythms in women with breast cancer before and after treatment: a 1-year longitudinal study. Support Care Cancer 2014;22(9):2535-45.

[25] Liu L, Fiorentino L, Natarajan L, et al. Pre-treatment symptom cluster in breast cancer patients is associated with worse sleep, fatigue and depression during chemotherapy. Psychooncology 2009;18(2):187-94.

[26] Smagula SF, Stone KL, Fabio A, et al. Risk factors for sleep disturbances in older adults: evidence from prospective studies. Sleep Med Rev 2016;25: 21-30.

[27] Colagiuri B, Christensen S, Jensen AB, et al. Prevalence and predictors of sleep difficulty in a national cohort of women with primary breast cancer three to four months postsurgery. J Pain Symptom Manage 2011;42(5):710-20.
[28] American Psychiatric Association. Diagnostic and statistical manual of mental disorders (DSM-V). Washington, DC: American Psychiatric Association; 2013.

[29] Carpenter JS, Johnson D, Wagner L, et al. Hot flashes and related outcomes in breast cancer survivors and matched comparison women. Oncol Nurs Forum 2002;29(3):E16-25.

[30] Rissling MB, Liu L, Natarajan L, et al. Relationship of menopausal status and climacteric symptoms to sleep in women undergoing chemotherapy. Support Care Cancer 2011;19(8):1107-15.

[31] Ho SY, Rohan KJ, Parent J, et al. A longitudinal study of depression, fatigue, and sleep disturbances as a symptom cluster in women with breast cancer. J Pain Symptom Manage 2015;49(4):707-15.

[32] Berger AM, Wielgus K, Hertzog M, et al. Patterns of circadian activity rhythms and their relationships with fatigue and anxiety/depression in women treated with breast cancer adjuvant chemotherapy. Support Care Cancer 2010;18(1): 105-14.

[33] Jung BF, Ahrendt GM, Oaklander AL, et al. Neuropathic pain following breast cancer surgery: proposed classification and research update. Pain 2003; 104(1-2):1-13.

[34] Jung BF, Herrmann D, Griggs J, et al. Neuropathic pain associated with nonsurgical treatment of breast cancer. Pain 2005;118(1-2):10-4.

[35] Cohen SP, Mao J. Neuropathic pain: mechanisms and their clinical implications. BMJ Br Med J 2014;348.

[36] Buysse DJ, Ancoli-Israel S, Edinger JD, et al. Recommendations for a standard research assessment of insomnia. Sleep 2006;29(9):1155-73. 